\title{
ERRATA
}

\section{Factors influencing time-location patterns and their impact on estimates of exposure: the Multi-Ethnic Study of Atherosclerosis and Air Pollution (MESA Air)}

Elizabeth W. Spalt, Cynthia L. Curl, Ryan W. Allen, Martin Cohen, Kayleen Williams, Jana A. Hirsch, Sara D. Adar and Joel D. Kaufman

Journal of Exposure Science and Environmental Epidemiology (2016) 26, 435; doi:10.1038/jes.2016.12

Correction to: Journal of Exposure Science and Environmental Epidemiology (2015); advance online publication 29 April 2015; doi:10.1038/jes.2015.26
The Supplementary Information published with the article was incomplete. The MESA Air Questionnaire was omitted. It can be accessed via the online version of this erratum.

Supplementary Information accompanies the paper on the Journal of Exposure Science and Environmental Epidemiology website (http:// www.nature.com/jes)

\section{Time-location patterns of a diverse population of older adults: the Multi-Ethnic Study of Atherosclerosis and Air Pollution (MESA Air)}

Elizabeth W. Spalt, Cynthia L. Curl, Ryan W. Allen, Martin Cohen, Sara D. Adar, Karen H. Stukovsky, Ed Avol, Cecilia Castro-Diehl, Cathy Nunn, Karen Mancera-Cuevas and Joel D. Kaufman

Journal of Exposure Science and Environmental Epidemiology (2016) 26, 435; doi:10.1038/jes.2016.13

Correction to: Journal of Exposure Science and Environmental Epidemiology (2015); advance online publication 29 April 2015; doi:10.1038/jes.2015.29
The Supplementary Information published with the article was incomplete. The MESA Air Questionnaire and the Time-Location Diary were omitted. These documents can be accessed via the online version of this erratum.

Supplementary Information accompanies the paper on the Journal of Exposure Science and Environmental Epidemiology website (http:// www.nature.com/jes) 